\title{
Modeling of Structural and Environmental Effects on Microelectromechanical (MEMS) Vibratory Gyroscopes
}

\author{
Md. Imrul Kaes, D.S-K Ting, Mohammed Jalal Ahamed \\ Micro/Nano-Mechatronics Laboratory, Mechanical, Automotive \& Material Engineering \\ University of Windsor, Windsor, ON, Canada \\ Email: jahamed@uwindsor.ca
}

\begin{abstract}
-
In this paper we investigate the effects of stiffness, damping and temperature on the performance of a MEMS vibratory gyroscope. The stiffness and damping parameters are chosen because they can be appropriately designed to synchronize the drive and sense mode resonance to enhance the sensitivity and stability of MEMS gyroscope. Our results show that increasing the drive axis stiffness by $50 \%$ reduces the sense mode amplitude by $\sim 27 \%$ and augments the resonance frequency by $\sim 21 \%$. The stiffness and damping are mildly sensitive to typical variations in operating temperature. The stiffness increases by $1.25 \%$, while the damping decreases by $3.81 \%$, when the temperature is raised from $0^{\circ} \mathrm{C}$ to $45^{\circ} \mathrm{C}$. Doubling the damping reduces the oscillation amplitude by $80 \%$, but $\sim 1 \%$ change in the frequency. The predicted effects of stiffness, damping and temperature can be utilized to design a gyroscope for the desired operating condition.
\end{abstract}

Keywords- MEMS gyroscope, Resonator, Spring Stiffness Coefficient, Damping Coefficient, Temperature, Coriolis

\section{INTRODUCTION}

Microelectromechanical systems (MEMS) based inertial sensors such as accelerometers and gyroscopes are the most commercially successful MEMS sensors to date. With the rapid advancement of semi-conductor based mass manufacturing, the cost, size and power consumption are continuously decreasing, enabling new applications in automotive, aerospace, biomedical and consumer electronics. MEMS vibratory gyroscopes are physical sensors that can detect and measure the angular motion of an object relative to an inertial frame of reference. MEMS vibratory sensors show promising vibration characteristics. Fabrication accuracies are comparable to macro-scale high performance sensors [1]-[4]. A vibratory gyroscope uses a vibrating structure (proof mass) to determine the rate of rotation [5]. The vibrating proof mass tends to vibrate along the same plane even if its support rotates. The Coriolis Effect causes the object to exert a force on its support, and the rate of rotation can be determined from the exerted force. The energy is transferred from the vibrating drive axis to the sense axis through Coriolis force. The sense mode response detects the angular velocity. The sensing performance of the MEMS gyroscope can deteriorate because of the influence of time-varying parameters such as damping, cross stiffness, and environmental variations. They generate a frequency of oscillation mismatch between the two vibrating axes [6]. Due to fabrication imperfections and environmental effects, true values of resonant frequencies deviate from their nominal values (which is known as frequency mismatching). These lead to considerable reduction in the sensitivity of the gyroscope. Therefore, it is necessary to design proper stiffness and damping for achieving a robust gyroscope.

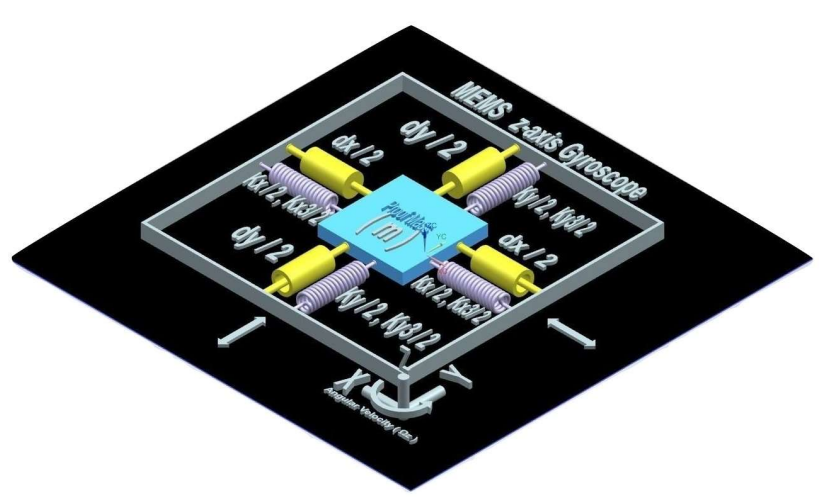

Figure 1. Schematic showing MEMS gyroscope model.

Optimization of time varying system parameters for better performance can be achieved by iterative modelling and simulation procedure. Various modeling techniques, including finite element analysis (FEA) modeling, analytical modeling and simplified lumped parameter modeling are employed [7]. FEA based modeling are robust, flexible and accurate in solving the complete multi-physics problem. Additionally, it can be coupled with controllers and electronics for selecting an appropriate controller [8].

Usually gyroscope displacement measurement varies under open loop scheme which can cause undesirable performance characteristics such as scale factor nonlinearity, limited dynamic range and narrow bandwidth [9]. In MEMS gyroscopes, 
manufacturing imperfection and noises often exist which negatively influence the resolution and performance. Errors due to noise are inevitable in actual MEMS gyroscopes, and thus, the controller has to be robust and effectively designed [10]. Postfabrication modeling of gyroscope using lumped-parameter techniques can shed light to the influence of system parameters, noise and environmental parameters on the gyro performance. This will allow iteration and design of a proper controller.

In this paper, a lumped-parameter model for predicting the gyroscope performance with the influence of various system parameters such as stiffness, damping and environmental condition (temperature) is analyzed. The results can be utilized to design a robust high performance gyroscope with efficient controller.

\section{MATHEMATICAL MODEL}

In a vibratory gyroscope the main sensing element is the proof mass, which is suspended above the substrate by several flexible beams. The overall dynamic system is usually modeled as a 2 degrees-of-freedom (2-DOF) spring-mass-damper system, as shown in Figure 1. The proof mass is suspended on the substrate using four springs (Figure 1), the other end of the springs are anchored to the substrate. An electrostatic actuation is used to give an oscillatory motion to the proof mass in the drive axis. When the proof mass is subject to an angular velocity, energy is transferred from drive axis to sense axis, causing it to oscillate. The mechanism for sensing position and velocity of the proof mass along the sense axis is present. Constant velocity of the proof mass and changing angular velocity of gyroscope about the $z$-axis are assumed. The MEMS vibratory gyroscope model includes proof mass $(m)$, drive axis displacement $(x)$, the angular velocity input $\left(\Omega_{\mathrm{z}}\right)$, and sense axis displacement $(y)$.

According to Lagrange's [11] equation, the dynamics of the gyroscope can be described by the equations of motion below [12]:

$\mathrm{m}_{\ddot{x}}+\mathrm{d}_{\mathrm{xx}} \dot{x}+\mathrm{k}_{\mathrm{xx}} \mathrm{x}+\mathrm{k}_{\mathrm{xy}} \mathrm{y}+\mathrm{d}_{\mathrm{xy}} \dot{y}=\mathrm{u}_{\mathrm{x}}+2 \mathrm{~m} \Omega_{\mathrm{z}} \dot{y}$
$\mathrm{~m} \ddot{y}+\mathrm{d}_{\mathrm{yy}} \dot{y}+\mathrm{k}_{\mathrm{yy}} \mathrm{y}+\mathrm{k}_{\mathrm{xy}} \mathrm{x}+\mathrm{d}_{\mathrm{xy}} \dot{x}=\mathrm{u}_{\mathrm{y}}+2 \mathrm{~m} \Omega_{\mathrm{z}} \dot{x}$

Manufacturing imperfections are translated mainly to the asymmetric spring and damping terms, $\mathrm{k}_{\mathrm{xy}}$ and $\mathrm{d}_{\mathrm{xy}}$. The drive and sense axes spring and damping terms $\mathrm{k}_{\mathrm{xx}}, \mathrm{k}_{\mathrm{yy}}, \mathrm{d}_{\mathrm{xx}}$ and $\mathrm{d}_{\mathrm{yy}}$ are mostly known, but have small unknown variations from their nominal values. The proof mass can be determined accurately, and $\mathrm{u}_{\mathrm{x}}, \mathrm{u}_{\mathrm{y}}$ are the control forces in the $\mathrm{x}$ and $\mathrm{y}$ directions.

Dividing gyroscope dynamics by the reference mass, the following equation can be obtained [13]:

$\ddot{q} *+\frac{D *}{m} q *+\frac{k 1 *}{m} \mathrm{q} *+\frac{k 3 *}{m}+\mathrm{q}^{* 3}$

$=\Omega^{*} \mathrm{q}^{*}+S^{*} * \mathrm{q}^{*}+2 \mathrm{~S}^{*} q^{*} *+\mathrm{u}^{*}$

where

$\mathrm{q}^{*}=\left[\begin{array}{l}x^{*} \\ y^{*}\end{array}\right], \mathrm{u}^{*}=\left[\begin{array}{l}u_{x^{*}} \\ u_{y^{*}}\end{array}\right], \mathrm{d}^{*}=\left[\begin{array}{cc}d_{x^{*}} & d_{x y^{*}} \\ d_{x y^{*}} & d_{y^{*}}\end{array}\right], \mathrm{k}_{1} *=\left[\begin{array}{cc}k_{x^{*}} & k_{x y^{*}} \\ k_{x y^{*}} & k_{y^{*}}\end{array}\right]$, $\mathrm{k}_{3} *=\left[\begin{array}{cc}k_{x^{3 *}} & 0 \\ 0 & k_{y^{3 *}}\end{array}\right], \mathrm{S} *=\left[\begin{array}{cc}0 & \Omega_{z^{*}} \\ -\Omega_{z^{*}} & 0\end{array}\right]$

Angular rate variation may be considered to be negligible. The equations of motion of the gyroscope are normalized to generalize the model. The final non-dimensional equation is derived in terms of normalized time, $t^{*}=\omega_{0} t$. With this, dividing both sides of the equation by the reference frequency and length gives [13]:

$\frac{\ddot{q}^{*}}{q_{0}}+\frac{d^{*}}{m \omega_{0}} \frac{\dot{q}^{*}}{q_{0}}+\frac{k_{1}^{*}}{m \omega_{0}^{2}} \frac{q^{*}}{q_{0}}+\frac{k_{3}^{*}}{m \omega_{0}^{2}} \frac{q^{* 3}}{q_{0}^{3}}$
$=\frac{\Omega_{z}^{*}}{\omega_{0}^{2}} \frac{\dot{q}^{*}}{q_{0}}+2 \frac{S^{*}}{\omega_{0}} \frac{\dot{q}^{*}}{q_{0}}+\frac{u^{*}}{m \omega_{0}^{2}} \frac{1}{q_{0}}$

where the parameters are defined as follows:

$q=\frac{q^{*}}{q_{0}}, d_{x y}=\frac{d_{x y}^{*}}{m \omega_{0}}, \Omega_{z}=\frac{\Omega_{z}^{*}}{\omega_{0}}, u_{x}=\frac{u_{x}^{*}}{m \omega_{0}^{2}} \frac{1}{q_{0}}, u_{y}=\frac{u_{y}^{*}}{m \omega_{0}^{2}} \frac{1}{q_{0}}$

$\omega_{x}=\sqrt{\frac{k_{x}}{m \omega_{0}^{2}}}, \omega_{y}=\sqrt{\frac{k_{y}}{m \omega_{0}^{2}}}, \omega_{x y}=\frac{k_{x y}}{m \omega_{0}^{2}}$,

$\delta_{x}=\frac{k_{x}^{3}}{m \omega_{0}^{2}} q_{0}^{2}, \delta_{y}=\frac{k_{y}^{3}}{m \omega_{0}^{2}} q_{0}^{2}$

Consequently, the non-dimensional representation becomes

$\ddot{q}+D \dot{q}+q k_{1}+k_{3} q^{3}=\Omega_{z}^{2} \dot{q}+2 S \dot{q}+u$

where

$q=\left[\begin{array}{l}x \\ y\end{array}\right], u=\left[\begin{array}{l}u_{x} \\ u_{y}\end{array}\right], D=\left[\begin{array}{cc}d_{x} & d_{x y} \\ d_{x y} & d_{y}\end{array}\right], k_{1}=\left[\begin{array}{cc}\omega_{x}^{2} & \omega_{x y} \\ \omega_{x y} & \omega_{y}^{2}\end{array}\right]$, $k_{3}=\left[\begin{array}{cc}\delta_{x} & 0 \\ 0 & \delta_{y}\end{array}\right], S=\left[\begin{array}{cc}0 & \Omega_{z} \\ -\Omega_{z} & 0\end{array}\right]$

Simulation is done to observe the influence of stiffness, damping and temperature on gyroscope performance to minimize time and cost of expensive trial and error with the actual fabrication cycle.

MATLAB Simulink [14] is used for simulation and computation. Spring stiffness, damping coefficient and temperature are known to have significant influence on the output performance of gyroscope. Thus, the objective of the simulation is to quantify the effects of these parameters. The spring stiffness and damping coefficient are varied from half to double of their nominal values. The temperature is varied from $0^{0} \mathrm{C}$ to $45^{0} \mathrm{C}$ to observe the temperature effect on stiffness, damping coefficient and on sensing magnitude. For the simulation model, the nominal values for the proof mass, stiffness, and damping are extracted from the literature reported in [12]. Proof mass, $\mathrm{m}=0.57 \mathrm{e}^{-8} \mathrm{~kg}$, damping coefficient along the drive-axis, $\mathrm{d}_{\mathrm{xx}}=0.429 \mathrm{e}^{-6} \mathrm{~N} \mathrm{~s} / \mathrm{m}$, damping error due to manufacturing imperfection, $\mathrm{d}_{\mathrm{xy}}=0.0429 \mathrm{e}^{-6} \mathrm{~N} \mathrm{~s} / \mathrm{m}$, damping coefficient along the sense-axis, $d_{y y}=0.687 \mathrm{e}^{-36} \mathrm{~N} \mathrm{~s} / \mathrm{m}$, spring stiffness along the drive-axis, $\mathrm{k}_{\mathrm{xx}}=80.98 \mathrm{~N} / \mathrm{m}$, stiffness error 
due to manufacturing imperfection, $\mathrm{k}_{\mathrm{xy}}=5 \mathrm{~N} / \mathrm{m}$, spring stiffness along the sense-axis, $\mathrm{k}_{\mathrm{yy}}=71.62 \mathrm{~N} / \mathrm{m}$, angular velocity, $\Omega_{\mathrm{z}}=5$ $\mathrm{rad} / \mathrm{s}$ are considered.

\section{MODEL RESULTS}

\section{A. Oscillation Frequency}

To identify the first resonance frequency, input frequency is varied, a sinusoidal electromagnetic force is introduced at the drive-axis and output is sensed both in drive and sense directions (Fig. 2). Usually the drive mode displacement is higher compared to sense mode due to the energy transfer.

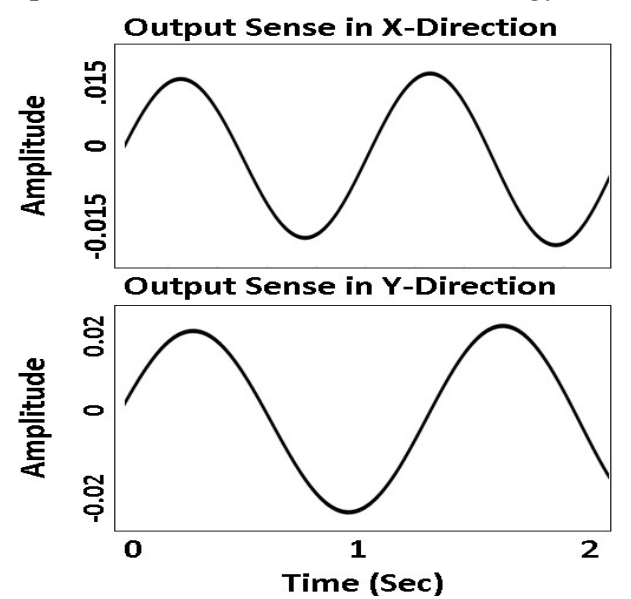

Figure 2. Displacement in drive mode (x) and sense mode (y) direction.

After sweeping the simulation with the frequency range of 1 $\mathrm{kHz}$ to $60 \mathrm{kHz}$ (Fig. 3), the resonance frequency is found at around $9.4 \mathrm{kHz}$. So, for better focusing, the frequency range is restricted from $10 \mathrm{kHz}-30 \mathrm{kHz}$ for other comparisons.

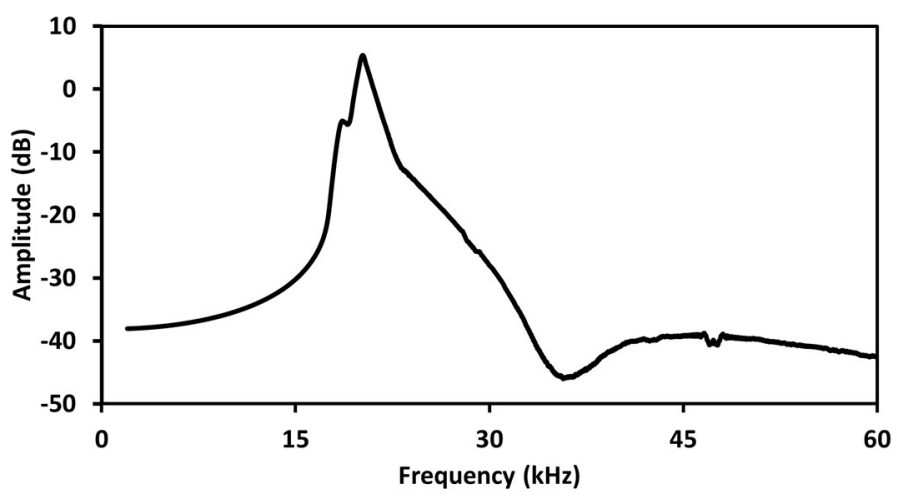

Figure 3. Frequency versus magnitude graph.

\section{B. Variation in Stiffness}

Suspension beam along drive and sense direction provides the stiffness necessary in each direction. Keeping the $\mathrm{k}_{\mathrm{yy}}$ value constant at $71.62 \mathrm{~N} / \mathrm{m}, \mathrm{k}_{\mathrm{xx}}$ is varied from $50 \%$ (normalized value 0.5 ) to $150 \%$ (normalized value 1.5 ). The normalized value of tuned reference stiffness $(80.98 \mathrm{~N} / \mathrm{m})$ is considered as 1. It is observed that magnitudes at resonance frequencies show linear decreasing trend (Fig. 4) and amplitudes decrease (Fig. 5) as stiffness increases. However, decreasing the stiffness creates phase lag and distortion in output response.

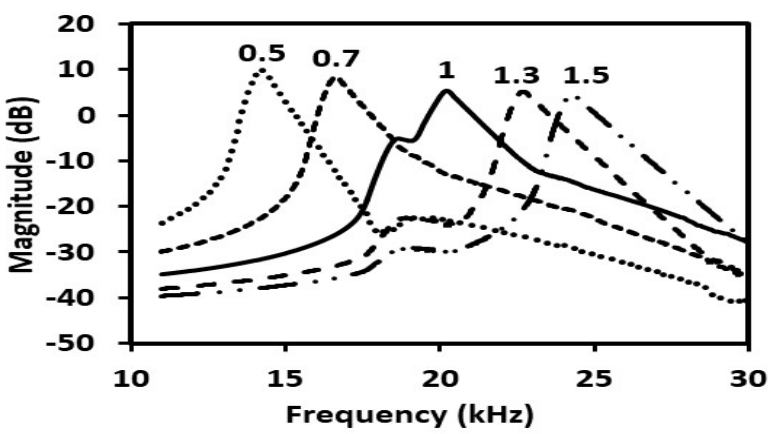

Figure 4. Effect of drive mode stiffness on resonance frequency and amplitude.

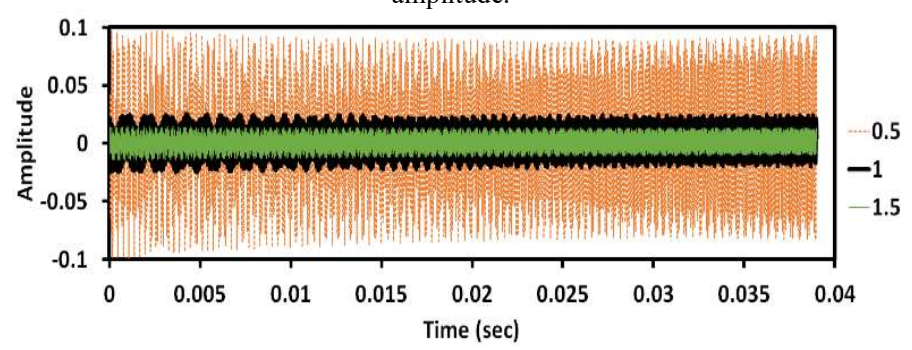

Figure 5. Effect of drive mode stiffness on output sense (sense mode stiffness is constant)

Keeping the $\mathrm{k}_{\mathrm{xx}}$ value constant, $\mathrm{k}_{\mathrm{yy}}$ is varied. It is observed that shift in resonance frequency is non-significant (Fig. 6).

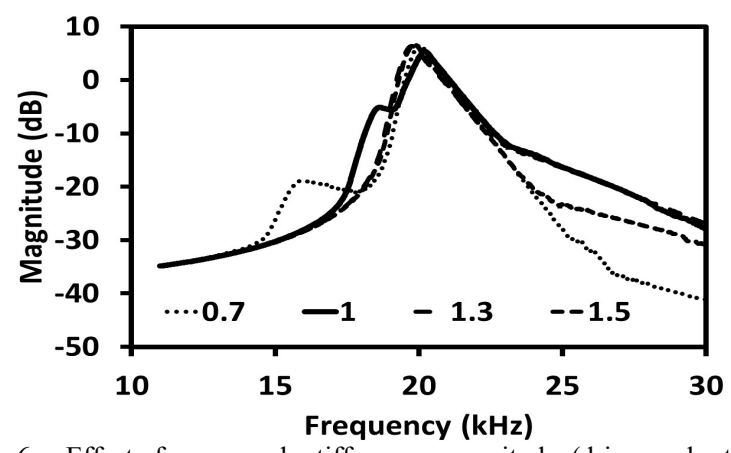

Figure 6. Effect of sense mode stiffness on magnitude (drive mode stiffness is constant).

Variation in stiffness $\left(\mathrm{k}_{\mathrm{xx}}\right.$ and $\left.\mathrm{k}_{\mathrm{yy}}\right)$ along both axes decreases magnitudes at resonance frequencies and resonance frequencies shift towards higher frequencies (Fig. 7) and amplitudes also decreases as stiffness increases (Fig. 8).

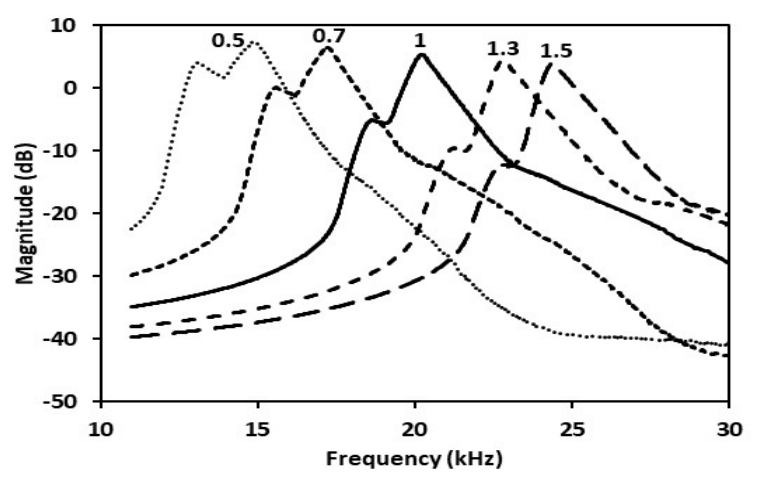

Figure 7. Combined effect of drive mode stiffness and sense mode stiffness on magnitude. 


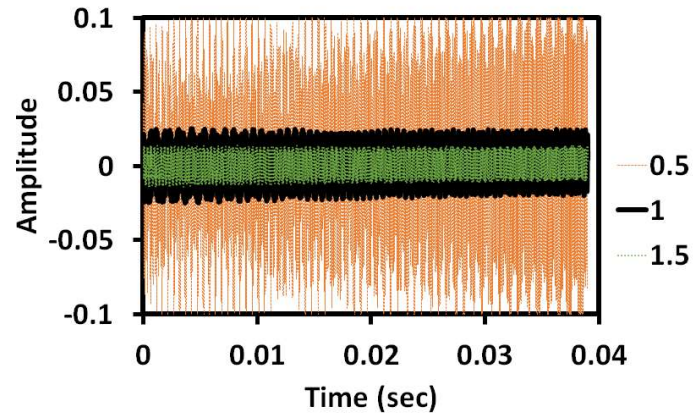

Figure 8. Effect of drive mode stiffness and sense mode stiffness on output sense.

\section{Variation in Damping}

Here, damping along drive axis is varied (Fig. 9). It is obvious from the figure that the resonance frequencies do not change that much but slope steepness decreases after resonance frequencies.

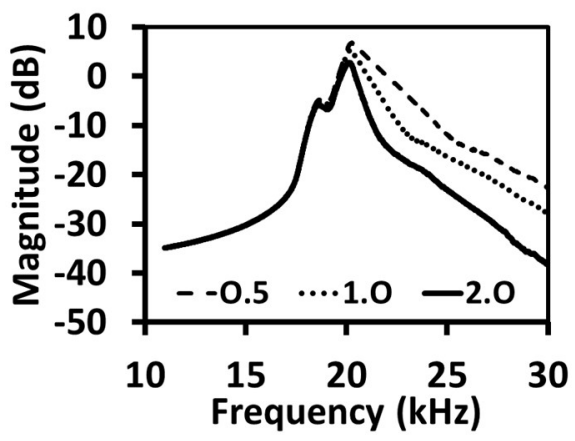

Figure 9. Effect of drive mode damping on magnitude.

\section{Temperature Variation}

One key disadvantage of MEMS gyrosocpe is its high thermal sensitivity. The frequency of oscillation drifts with temperture. Temperature variation mainly affects the stiffness and damping of the supporting beams. Here investigation on the temperature sensitivity for stiffness and damping is done. The variation of stiffness with temperature can be modeled using simplified linear relationship of [7] $K(T)=K_{0}(1-k \Delta T)$, where $K_{0}$ is the stiffness coefficient at reference temperature $300 \mathrm{~K}$ and $k=70$ ppm.

Variation of damping coefficient with temperature can be modeled as [7] $d(T)=d_{0} \cdot 1.28^{\ln \left(\frac{T}{300}\right)}$, where $\mathrm{T}$ is temperature in $\mathrm{K}, d_{0}$ is demping coefficient at reference temperature of 300 $K$. Magnitude shows positive increasing trend with the increase of temperature when stiffness and temperature relationship shows linear behaviour. The damping coefficient within industrial temperature range shows decreasing trend as temperature increases (Fig. 10, 11).

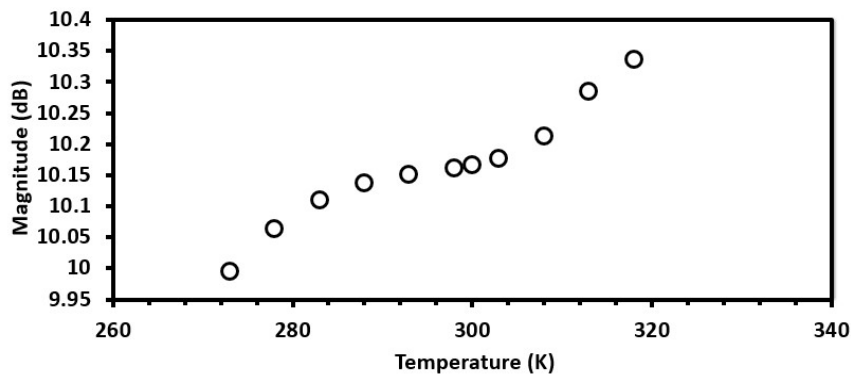

Figure 10. Effect of temperature on linear drive mode stiffness and magnitude

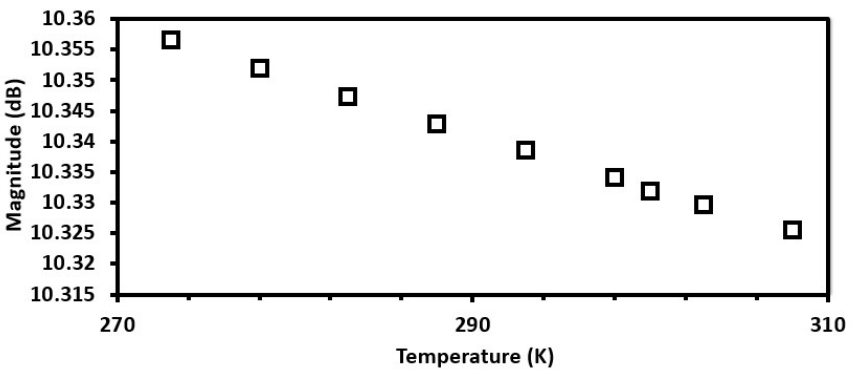

Figure 11. Effect of temperature on non-linear damping and magnitude

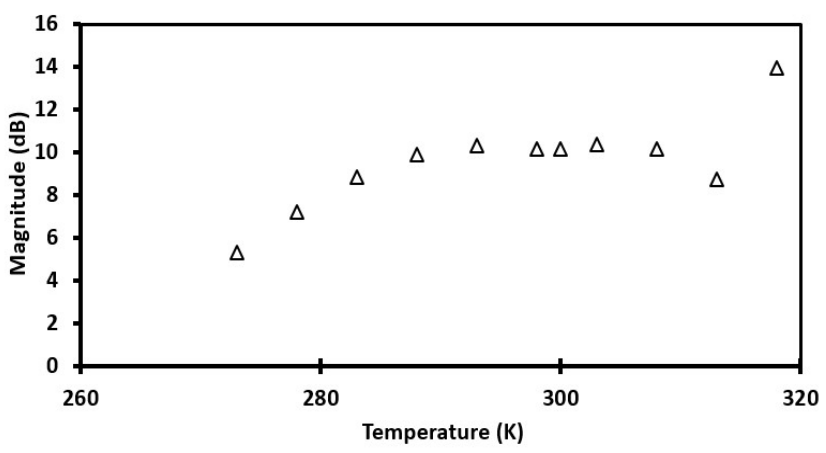

Figure 12. Effect of temperature on non-linear drive mode stiffness and magnitude.

The spring stiffness and temperature relationship shows nonliner behavior, the magnitude increases non-linearly up to a certain point before decreasing. At the end of the industrial temperature range, the magnitude increases drastically (Fig. 12)

\section{CONCLUSION AND FUTURE WORK}

This paper investigates the effect of variation of suspending stiffness coefficient, damping coefficient and temperature on the performance of MEMS gyroscope. Simulation results show how these parameters affect the error i.e. mismatch between the input and sense signals. This will help to design appropriate system parameters and controllers to increase gyroscope accuracy. Simulation results also show that stiffness is the dominant gyroscope parameter. The temperature can negatively impact resonance frequency and sense magnitude. In the future, we plan to extend this by including experimental validation of our results with MEMS gyroscopes and implementing a controller to minimize these effects and maximize the gyroscope performance. 


\section{ACKNOWLEDGEMENT}

The authors would like to thank the Natural Sciences and Engineering Research Council of Canada (NSERC) and University of Windsor for financial support.

\section{REFERENCES}

[1] M. J. Ahamed, D. Senkal, S. Member, A. A. Trusov, and A. M. Shkel, "Study of High Aspect Ratio NLD Plasma Etching and Postprocessing of Fused Silica and Borosilicate Glass," $J$. microelectromechanical Syst., vol. 24, no. 4, pp. 790-800, 2015.

[2] J. Liu, J. Jaekel, D. Ramdani, N. Khan, D. S.-K. Ting, and M. J. Ahamed, "Effect of Geometric and Material Properties on Thermoelastic Damping (TED) of 3D Hemispherical Inertial Resonator," in ASME International Mechanical Engineering Congress and Exposition, 2016, vol. 10, pp. 1-6.

[3] A. M. Shkel, M. Ahamed, and D. Senkal, "Method of fabricating micro-glassblown gyroscopes," US 9,702,728 B2, 2017.

[4] D. Senkal, M. J. Ahamed, M. H. A. Ardakani, S. Askari, and A. M. Shkel, "Demonstration of 1 million Q -factor on microglassblown wineglass resonators with out-of-plane electrostatic transduction," $J$ Microelectromechanical Syst., vol. 24, no. 1, pp. 29-37, 2015.

[5] F. Braghin, F. Resta, E. Leo, and G. Spinolab, "Nonlinear dynamics of vibrating MEMS," Sensors Actuators A Phys., vol. 134, no. 1, pp. 98-108, 2007.

[6] J. Fei and C. Batur, "A novel adaptive sliding mode control with application to MEMS gyroscope," ISA Trans., vol. 48, no. 1, pp. 7378,2009 .

[7] M. Wen et al., "Modeling and analysis of temperature effect on MEMS gyroscope," Electron. Components Technol. Conf., pp. 2048-2052, 2014.

[8] J. Wang, S. Ban, and Y. Yang, "A Differential Self-Integration DDot Voltage Sensor and Experimental Research," IEEE Sens. J., vol. 15 , no. 7 , pp. $3846-3852,2015$.

[9] W. T. Sung, S. Sung, J. G. Lee, and T. Kang, "Design and performance test of a MEMS vibratory gyroscope with a novel AGC force rebalance control," J. Micromechanics Microengineering, vol. 17, no. 10, pp. 1939-1948, 2007.

[10] W. Juan and J. Fei, "Adaptive fuzzy approach for non-linearity compensation in MEMS gyroscope," Trans. Inst. Meas. Control, vol. 35 , no. 8, pp. 1008-1015, 2013.

[11] R. G. Albrecht Bertram, Solid Mechanics: Theory, Modeling, and Problems, First Edit. Sachsen-Anhalt, Germany: Springer, 2013.

[12] J. Fei, W. Dai, M. Hua, and Y. Xue, "System dynamics and adaptive control of MEMS gyroscope sensor," IFAC Proc., vol. 44, no. 1, pp. 3551-3556, 2011.

[13] M. R. Moghanni, J. Keighobadi, and A. Ghanbari, "Fuzzy adaptive sliding mode controller for MEMS vibratory rate gyroscope," IFAC Proc., vol. 44, no. 1, pp. 4192-4197, 2011.

[14] Simulink, "Mathworks," 2018. [Online]. Available: https://www.mathworks.com/products/simulink.html. [Accessed: 26-Mar-2018]. 